\title{
Pharmacovigilance: towards a better understanding of the benefit to risk ratio
}

\section{S Simon}

$\mathrm{P}$ harmacovigilance is a critical component for determining the benefit to risk ratio of treatment. The potential for drug toxicity is determined throughout the lifetime of use of a drug or biological agent, including the development cycle. This includes both the preclinical as well as clinical data. Preclinical data are rarely extensive for biological therapies because it is difficult to study these drugs in animal models, whereas pharmacotoxicology can be very informative in the development of new drugs.

Toxic events caused by drugs or biological therapies may be identified at any time during treatment, as there is no absolutely safe treatment. There are certain patterns to the onset of these potential events. One pattern of onset for some treatments is early onset. In this situation, if patients continue to take the drug, the incidence of the toxic event may decrease over time. Other toxic events might be delayed and only noted with continued use of a drug. There is a third possible pattern-namely, treatment that induces a chronic but constant event, such as the use of non-selective non-steroidal anti-inflammatory drugs, which are believed to cause one or two gastric ulcers a month over the lifetime of exposure.

Toxic side effects may be noted in clinical trial reports as adverse events (AEs), serious adverse events (SAEs) or as clinically relevant adverse events (CRAEs). In a regulatory environment, at least in the USA, we are interested in all types of AEs. The information obtained is included on the product label which is used to inform both patients and their doctors about the nuances in the use of treatments. The label is constantly updated so that information gained in postmarketing of a product is constantly reviewed and consideration given to adding this newly acquired information to the product label. SAEs, once noted, may actually lead to very specific identified warnings on the product labels or even withdrawal of the drug.

Although we usually obtain experience about the potential risk of a new drug in randomised controlled clinical studies, which may lead to regulatory approval for use, these studies tend to be relatively short and unique. They often consist of a highly restricted group of patients who do not necessarily reflect the standard population who will use the drug (younger, healthier patients who are not required to use multiple other drugs). Furthermore, studies such as these are of variable length, rarely lasting longer than six months to one year. For rheumatology patients who may have syndromes or diseases which last for 30 years to a lifetime, these observed responses occupy rather a short period of time. Accumulated information from these trials will typically inform the regulator as well as the clinician about the more common possible toxic effects, but more rare events may not be encountered at all. Unfortunately, phase IV studies, unless unusually large, often do not reflect potential rare events either. Even by adhering to the International Committee on Harmonisation (ICH) guidelines for the extent of exposure to an experimental product, it is unlikely a rare event will be noted.

Various methods of discovering these more rare toxic events exist-for example, determination of data from prolonged long term controlled clinical trials, observational epidemiological studies (including case-control, cohort, and cross sectional analyses), demographic methods, drug use surveys, voluntary spontaneous reports, automated databases linking drugs and disease, as well as early established registries. ${ }^{1-3}$

At present, in the USA, we often rely on voluntary spontaneous reporting of AEs, usually by the clinician in everyday use of the drug after approval. Unfortunately, this reporting system is often afflicted by the Weber effect. ${ }^{45}$ Fletcher compared two types of trial and showed that there was $98 \%$ underreporting in the spontaneous reporting system in comparison with event monitoring, such as in a large longitudinal cohort or registry programme. ${ }^{6}$ This "effect" showed that the busy clinician tends to report events with new treatments but does not continue do this as religiously after the first two years of marketing. Thus, there is an imbalance in the toxicity described in such reports. Older drugs, which may possess unique toxicities, are thought to be better understood as they have been around for some time and, therefore, clinicians feel they do not need to report AEs.

When the Federal Drug Association (FDA) becomes aware that a drug has potentially important toxicity (through primary or secondary reviews, spontaneous reporting, or by an outside group through a citizen's petition) it thoroughly reviews the original new drug application (NDA) database which was used for the initial approval of the putative drug. At the same time a formal analysis is started by reviewing all the spontaneous reports accumulated by the Adverse Events Reporting System through "Medwatch" (this is primarily performed by the Office of Drug Safety). Additionally, the sponsor of the drug is required to review its safety database and to ensure that the FDA is aware of all worldwide events. In some circumstances, further studies are designed to answer specific questions about the toxic effect in question. In sum, there is an attempt to accumulate the best available evidence to provide information about the safety of the product as it is generally used (benefit to risk ratio).

Thus, pharmacovigilance may be the only way that we can determine rare toxic events. Yet in the USA we depend on a predominantly voluntary system to provide this information. To maximise the benefits of this system will require new education for the busy clinician to rekindle their enthusiasm for the exercise. Furthermore, more resources will be needed to allow the developers of these drugs to pursue a robust programme to ensure the reporting of these events even when a drug is older than two years, let alone available for 30 years.

\section{Author's affiliation}

L S Simon, Division of Analgesic, Anti-inflammatory and Ophthalmologic Drug Products, Center for Drug Evaluation and Research ODEV; Food and Drug Administration; Harvard Medical School

Correspondence to: Dr L S Simon, 9201 Corporated Blvd, Room N-314, HFD-550, ODEV, CDER, FDA, Rockville, MD 20850, USA simon $1 @ c d e r . f d a . g o v$ 


\section{REFERENCES}

1 Edlavitch SA. Post marketing surveillance methodologies. Drug Intell Clin Pharm 1988;22:68-78.

2 Shapiro S. The epidemiological evaluation of drugs. Acta Med Scand Suppl 1984;683:23-7.

3 Strom BL. Overview of different logistical approaches to post marketing surveillance. J Rheumatol Suppl 1988;17:9-13.
4 Leiper JM, Lawson DH. Why do doctors not report adverse drug reactions? Neth J Med 1985;28:546-50.

5 Strom BL, Melmon KL, Miettinen OS. Post marketing studies of drug efficacy. Arch Intern Med 1985;145:1791-4.

6 Fletcher AP. Spontaneous adverse drug reaction reporting vs event monitoring: a comparison. J R Soc Med 1991;84:341-4.

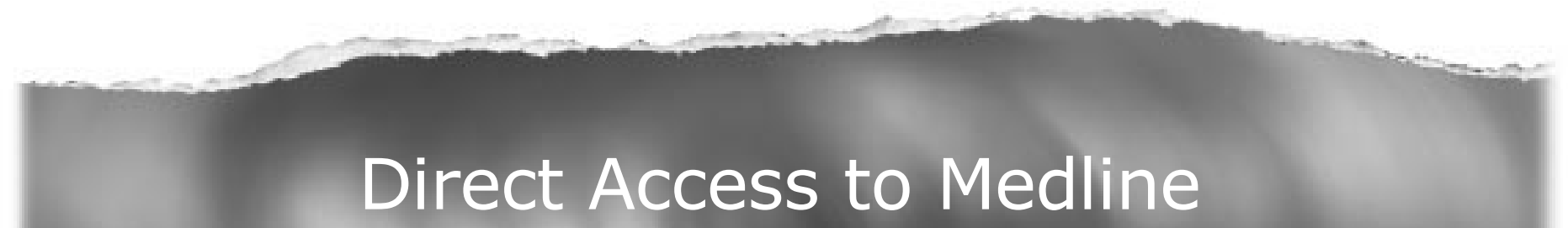

\section{Medline}

Link to Medline from the homepage and get straight into the National Library of Medicine's premier bibliographic database. Medline allows you to search across 9 million records of bibliographic citations and author abstracts from approximately 3,900 current biomedical journals.

www.annrheumdis.com 